\title{
Energy Harvesting Estimation from the Vibration of a Simply Supported Beam
}

\author{
Aviral Rajora \\ Delft University of Technology, Netherlands
}

\author{
Ajit Dwivedi \\ Steel Authority of India Limited, Bhilai, India
}

Ankit Vyas

Ministry of Health and Family Welfare, New Delhi, India

\section{Satyam Gupta}

Infosys Limited, Mysore, India

\author{
Amit Tyagi \\ Indian Institute of Technology (Banaras Hindu University), Varanasi, India
}

(Received 1 November 2014; accepted 19 April 2016)

Vibration-based energy harvesting has been investigated in this paper with the goal to utilize the ambient vibration energy to power small electronic components by converting vibration energy into electrical energy. A simply supported beam with a bonded high density piezoelectric patch to the surface is considered for the analysis. Analytical model for free vibration analysis is developed by starting with the linear constitutive relations for the beam and the patch. The equation of motion for transverse vibration of the beam is developed by considering the elastic as well as electrical properties in the generalized Hookes law and accordingly a transverse displacement function satisfying the simply supported boundary conditions is used for achieving the modal frequencies. Additionally, an analytical model is developed in order to estimate the energy generated under the action of a harmonic force applied on the surface of the patch. The results of the analytical model are validated using simulation software ANSYS and COMSOL.

The developed analytical model is used to study the behavior of a simply supported harvester with various patch dimensions and locations. This paper throws light on parametric studies of eigen frequencies as well as extracted power corresponding to operating conditions.

\section{NOMENCLATURE}

$D, \quad$ Electric charge density displacement matrix;

$e, \quad$ Coupling coefficient for stress-charge form;

$\epsilon, \quad$ Strain;

p, $\quad$ Electric permittivity;

$E_{z}, \quad$ Electric Field ;

$M(x, t)$, Moment on the beam at location $\mathrm{x}$, time $\mathrm{t}$;

$F$, Applied Force;

$\rho_{1}, \quad$ Density of the material 1 ;

$\rho_{2}, \quad$ Density of the material 2;

$E_{1}, \quad$ Youngs modulus of material 1;

$E_{2}, \quad$ Youngs modulus of material 2;

$l$, Length of the beam;

$b-a, \quad$ Length of patch starting at location $\mathrm{a}$;

$h_{1}$, Height of the beam;

$h_{2}$, Height of the patch;

$C$, Damping coefficient;

$K, \quad$ Stiffness Matrix;
$M, \quad$ Mass Matrix;

$F_{0}$, Applied force;

$V_{0}$, Voltage across the load resistance;

$C_{p}$, Capacitance of the energy harvesting circuit;

$R, \quad$ Load Resistance for energy harvesting circuit;

$P$, Power Generated.

\section{INTRODUCTION}

The growing demand for energy in various sectors has motivated researchers to look into alternative forms of energy generation at both large and small scales. Various devices have become miniature with the advancement of nanotechno- logy. However, this decrease in size is limited due to sizeable batteries. Thus, it is becoming essential to find a way to replace bulky conventional batteries in order to facilitate developing micro-electronic mechanical devices. Roundy et al. ${ }^{1}$ has shown a comparison of power scavenging from various energy sources like vibrations, solar, and various chemical batteries 
and found that ambient vibrations are a potential source of energy for the applications where continuous power is desired with long life. Miniature wireless electronic devices require a very low output power; ambient vibration energy can be used to power these devices. In light of this, our attention is focused on vibration energy harvesting.

There are different transduction mechanisms for converting vibration energy into useful electrical energy. These are piezoelectric, ${ }^{1}$ electromagnetic, ${ }^{2}$ and electrostatic. ${ }^{3}$ The comparison between maximum energy densities of these three transducers is given in Table $1 .{ }^{4}$ In the present study, piezoelectric materials have been used because they have relatively simple configurations and a high conversion efficiency.

Various work has been done in the past decade for estimating the vibration energy harvested using piezoelectric material. The effect of piezoelectricity on the elasticity is considered by applying a constitutive relation, as discussed by Tyagi and Ghosh. ${ }^{5}$ Sodano et al. ${ }^{6}$ presented the review of the research that has been performed in the area of power harvesting and the future goals that must be achieved for power harvesting systems to find their way into everyday use. In another paper, the same authors ${ }^{7}$ developed a model of the PZT power harvesting device, which simplifies the design procedure necessary for determining the appropriate size and vibration levels necessary for sufficient energy to be produced and supplied to the electronic devices. Chen et al. ${ }^{8}$ proposed a novel piezoelectric cantilever bimorph micro transducer electro-mechanical energy conversion model both analytically and experimentally. They noted that the vibration induced voltage is inversely proportional to the length of the cantilever beam. The review article by Priya ${ }^{9}$ provides a thorough review of developments in the area of piezoelectric energy harvesting. Erturk and Inman ${ }^{10}$ presented the exact analytical solution of a cantilevered piezoelectric energy harvester with Euler-Bernoulli beam assumptions. Xu et al. ${ }^{11}$ experimentally studied a high performance bi-stable piezoelectric harvester based on simply supported buckled beam. Erturk and Inman ${ }^{12}$ evaluated the performance of the bimorph device extensively for the short circuit and open circuit resonance frequency excitations and the accuracy of the model has been shown in all the cases.

It is generally accepted that a simplified analytical model for a simply supported beam will help understand the basic physics behind working of piezoelectric energy harvester. In the present work an analytical model is developed for calculating the modal frequencies using the Euler-Bernoulli Beam Equation. An analytical model is also developed for calculation of energy harvested by the piezoelectric patch bonded to a simply supported beam and the results are verified using ANSYS and COMSOL.

\section{FREE VIBRATION ANALYSIS}

A simply supported beam with thickness $h_{1}$ with an elastic patch of thickness $h_{2}$ that is centrally bonded to the beam surface is considered for the analysis.

The linear constitutive relation for the beam with piezoelectric patch is given by:

$$
D=e_{13} \varepsilon_{x x}+p_{33} E_{z}
$$

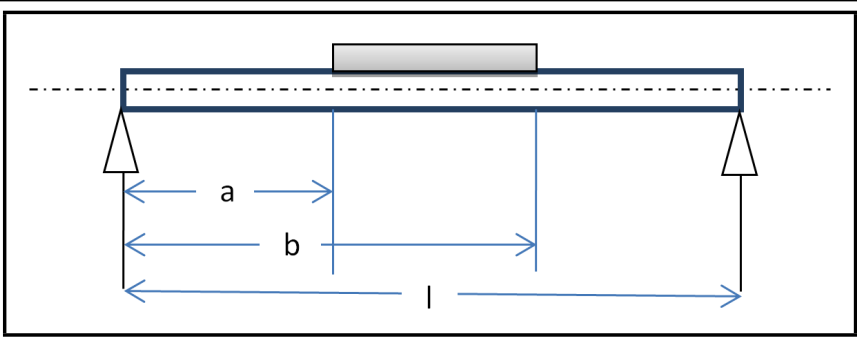

Figure 1. Reference figure for theoretical analysis.

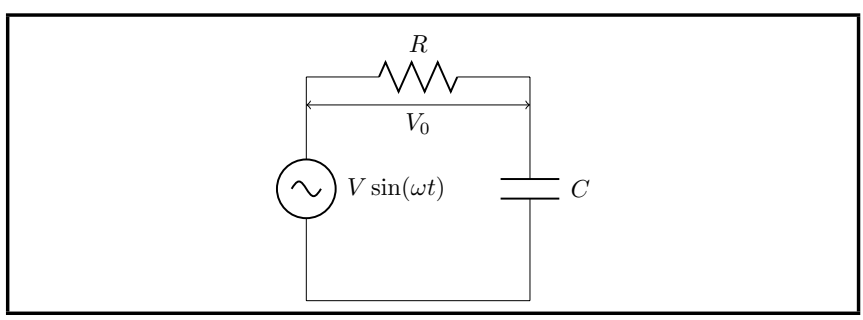

Figure 2. Circuit Diagram for Analysis.

Since there is no applied voltage, i.e., $D=0$, from Eq. (1), we get:

$$
E_{z}=-\left[\frac{e_{13} \varepsilon_{x x}}{p_{33}}\right]
$$

And we know that:

$$
\varepsilon_{x x}=\frac{d u}{d x}=-z\left(\frac{d^{2} w}{d x^{2}}\right) .
$$

So finally,

$$
E_{z}=\frac{z e_{13}\left(\frac{d^{2} w}{d x^{2}}\right)}{p_{33}} .
$$

According to Euler-Bernoulli beam theory:

$$
M(x, t)=E I(x)\left[\frac{\partial^{2} w(x, t)}{\partial x^{2}}\right] .
$$

And equilibrium equations are:

$$
\frac{\partial N_{x}}{\partial x}=0 ; \quad \frac{\partial^{2} M_{x}}{d x^{2}}-\rho h\left[\frac{\partial^{2} w(x, t)}{d t^{2}}\right]=0 .
$$

Hooke's law:

$$
\begin{array}{r}
\sigma_{x, 1}=E_{1} \varepsilon_{x x}(\text { For beam }) ; \\
\sigma_{x, 2}=E_{2} \varepsilon_{x x}-e_{13} E_{z}(\text { For patch }) .
\end{array}
$$

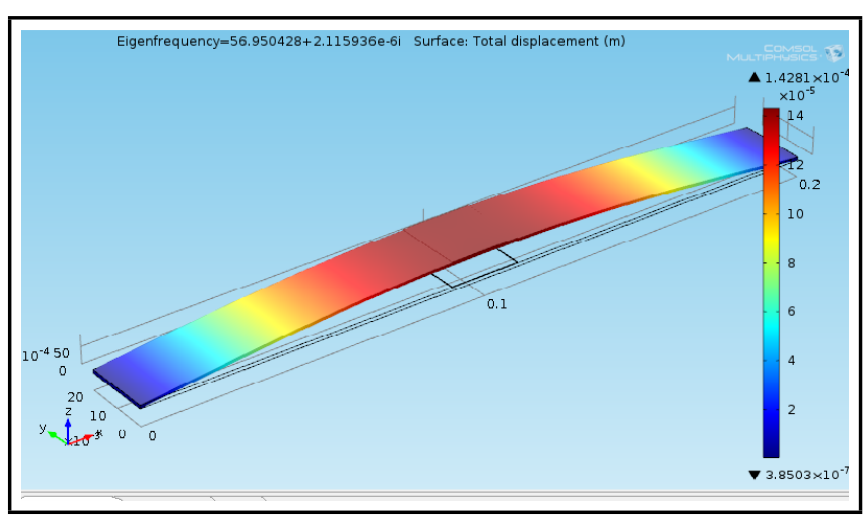

Figure 3. Simply supported beam vibrating in mode I. 
It is assumed that the patch is perfectly bonded to the beam and contributes to the force and moment. The equilibrium Eq. (6) for the force and moment are written as:

$$
\frac{\partial N_{x}}{\partial x}=0 ; \quad \frac{\partial^{2} M_{x}}{d x^{2}}-\rho h\left[\frac{\partial^{2} w(x, t)}{d t^{2}}\right]=0 ;
$$

where, $N_{x}=\int \sigma_{x, 1} d z+\int \sigma_{x, 2} R_{1} d z, M_{x}=\int \sigma_{x, 1} z d z+$ $\int \sigma_{x, 2} R_{1} z d z$, and $\rho h=\rho_{1} h_{1}+R_{1} \rho_{2} h_{2}$.

Where $R_{1}$ is a location function which can be expressed in terms of a Heaviside function:

$$
R_{1}=\left\{\begin{array}{ll}
0 ; & \text { when } \quad x<a \text { or } x>b \\
1 ; & \text { when } \quad a \leq x \leq b
\end{array} .\right.
$$

Therefore, from equation Eq. (7) and (8),

$M_{x}=\int_{\frac{-h_{1}}{2}}^{\frac{h_{1}}{2}} E_{1} \varepsilon_{x x} z \partial z+\int_{\frac{h_{1}}{2}}^{\frac{h_{1}+2 h_{2}}{2}}\left(E_{2} \varepsilon_{x x}+e_{13} \frac{e_{13} \varepsilon_{x x}}{p_{33}}\right) R_{1} z \partial z$.

Now from equation Eq. (2), $\varepsilon_{x x}=-z \frac{\partial^{2} w}{\partial x^{2}}$.

Therefore,

$$
\begin{aligned}
M_{x}= & -\int_{-\frac{h_{1}}{2}}^{\frac{h_{1}}{2}} E_{1} z^{2} \frac{\partial^{2} w}{\partial x^{2}} \partial z \\
& -\frac{\int_{\frac{h_{1}+2 h_{2}}{2}}^{2}}{\frac{h_{1}}{2}}\left(E_{2}+\frac{e_{13}{ }^{2}}{p_{33}}\right) R_{1} z^{2} \frac{\partial^{2} w}{\partial x^{2}} \partial z ; \\
M_{x}= & -E_{1} \frac{\partial^{2} w}{\partial x^{2}}\left(\frac{h_{1}^{3}}{12}\right) \\
& -\left(E_{2}+\frac{e_{13}{ }^{2}}{p_{33}}\right) R_{1} \frac{\partial^{2} w}{\partial x^{2}}\left(\frac{\left(h_{1}+2 h_{2}\right)^{3}-h_{1}{ }^{3}}{24}\right) ; \\
M_{x}= & -D_{1} \frac{\partial^{2} w}{\partial x^{2}}-D_{2} R_{1} \frac{\partial^{2} w}{\partial x^{2}} ;
\end{aligned}
$$

where, $D_{1}=\frac{E_{1} h_{1}^{3}}{12} ; D_{2}=\left(E_{2}+\frac{e_{13}{ }^{2}}{p_{33}}\right)\left(\frac{\left(h_{1}+2 h_{2}\right)^{3}-h_{1}{ }^{3}}{24}\right)$. Differentiating equation Eq. (10) with respect to $x$, we get:

$$
\begin{aligned}
\frac{\partial M_{x}}{\partial x}= & -D_{1} \frac{\partial^{3} w}{\partial x^{3}}-D_{2}\left[\frac{\partial R_{1}}{\partial x} \frac{\partial^{2} w}{\partial x^{2}}+R_{1} \frac{\partial^{3} w}{\partial x^{3}}\right] \\
\frac{\partial^{2} M_{x}}{\partial x^{2}}= & -D_{1} \frac{\partial^{4} w}{\partial x^{4}} \\
& -D_{2}\left[\frac{\partial^{2} R_{1}}{\partial x^{2}} \frac{\partial^{2} w}{\partial x^{2}}+2 \frac{\partial R_{1}}{\partial x} \frac{\partial^{3} w}{\partial x^{3}}+R_{1} \frac{\partial^{4} w}{\partial x^{4}}\right] .
\end{aligned}
$$

Putting this value of $\frac{\partial^{2} M_{x}}{\partial x^{2}}$ in equation Eq. (6), we get:

$$
\begin{array}{r}
-D_{1} \frac{\partial^{4} w}{\partial x^{4}}-D_{2}\left[\frac{\partial^{2} R_{1}}{\partial x^{2}} \frac{\partial^{2} w}{\partial x^{2}}+2 \frac{\partial R_{1}}{\partial x} \frac{\partial^{3} w}{\partial x^{3}}+R_{1} \frac{\partial^{4} w}{\partial x^{4}}\right]= \\
\rho h \frac{\partial^{2} w}{\partial t^{2}} .
\end{array}
$$

Boundary conditions for the simply supported beam are:

$$
\begin{aligned}
& x=0 ; \quad w=0 ; \quad \frac{\partial^{2} w}{\partial x^{2}}=0 ; \\
& x=l ; \quad w=0 ; \quad \frac{\partial^{2} w}{\partial x^{2}}=0 .
\end{aligned}
$$

To seek a solution, the following transverse displacement function is used for the simply supported beam:

$$
w=\sum A_{m} \sin \left(\frac{m \pi x}{l}\right) e^{i \omega t} .
$$

Putting w from Eq. (12) into Eq. (11), we get:

$$
\begin{gathered}
\left(-D_{1} \frac{\pi^{4}}{l^{4}}-D_{2} \frac{\pi^{4}}{l^{4}} R_{1}\right) \sum^{A_{m}} m^{4} \sin \left(\frac{m \pi x}{l}\right) \\
+D_{2} \frac{\pi^{2}}{l^{2}} \sum^{A_{m}} m^{2} \sin \left(\frac{m \pi x}{l}\right) \frac{\partial^{2} R_{1}}{\partial x^{2}} \\
-2 D_{2} \frac{\pi^{3}}{l^{3}} \sum^{A_{m}} m^{3} \cos \left(\frac{m \pi x}{l}\right) \frac{\partial R_{1}}{\partial x}= \\
\omega^{2} \rho h \sum^{A_{m}} \sin \left(\frac{m \pi x}{l}\right) .
\end{gathered}
$$

To obtain the modal solution, both the sides of equation Eq. (13) are multiplied by and integrated over the length $l$ of the host beam.

$$
\begin{gathered}
-D_{1} \frac{\pi^{4}}{l^{4}} \int_{0}^{l} \sum^{A_{m}} m^{4} \sin \left(\frac{m \pi x}{l}\right) \sin \left(\frac{p \pi x}{l}\right) d x \\
-D_{2} \frac{\pi^{4}}{l^{4}} \int_{0}^{l} R_{1} \sum^{A_{m}} m^{4} \sin \left(\frac{m \pi x}{l}\right) \sin \left(\frac{p \pi x}{l}\right) d x \\
+D_{2} \frac{\pi^{2}}{l^{2}} \int_{0}^{l} \frac{\partial^{2} R_{1}}{\partial x^{2}} \sum^{A_{m}} m^{2} \sin \left(\frac{m \pi x}{l}\right) \sin \left(\frac{p \pi x}{l}\right) d x \\
-2 D_{2} \frac{\pi^{3}}{l^{3}} \int_{0}^{l} \frac{\partial R_{1}}{\partial x} \sum^{A_{m}} m^{3} \sin \left(\frac{m \pi x}{l}\right) \sin \left(\frac{p \pi x}{l}\right) d x= \\
\omega^{2} \rho h \int_{0}^{l} \sum^{A_{m}} \sin \left(\frac{m \pi x}{l}\right) \sin \left(\frac{p \pi x}{l}\right) d x
\end{gathered}
$$

We make use of following mathematical relations:

$$
\text { if } R= \begin{cases}0 ; & \text { when } \quad x<a \text { or } x>b \\ 1 ; & \text { when } \quad a \leq x \leq b\end{cases}
$$

$$
\begin{aligned}
& \text { then } \begin{array}{r}
\int_{a}^{b} \frac{d R}{d x} f(x) d x= \\
\int_{-\infty}^{\infty}\{\delta(x-b)-\delta(x-a)\} f(x) d x= \\
f(b)-f(a) ; \\
\int_{a}^{b} \frac{d^{2} R}{d x^{2}}= \\
\int_{-\infty}^{\infty}\left\{\delta^{\prime}(x-b)-\delta^{\prime}(x-a)\right\} f(x) d x= \\
f^{\prime}(a)-f^{\prime}(b) .
\end{array}
\end{aligned}
$$

Upon solving equation Eq. (14), we get Eq. (15).

Equation (15) is solved using MATLAB to get the modal frequencies. The results are verified using modeling in ANSYS and COMSOL software. The results obtained are discussed later in this paper.

\section{ENERGY HARVESTING}

The moment equation considering the externally applied distributed force on the patch area is given as: 


$$
\begin{gathered}
-D_{2} \frac{\pi^{4}}{l^{4}} \sum^{A_{m}} m^{4}\left[\left\{\frac{\sin \left(\frac{(m-p) \pi b}{l}\right)-\sin \left(\frac{(m-p) \pi a}{l}\right)}{2 \pi(m-p)}\right\}-\left\{\frac{\sin \left(\frac{(m+p) \pi b}{l}\right)-\sin \left(\frac{(m+p) \pi a}{l}\right)}{2 \pi(m+p)}\right\}\right] \\
-D_{2} \frac{\pi^{3}}{l^{3}} \sum^{A_{m}} m^{2}\left[\begin{array}{c}
p\left\{\sin \left(\frac{m \pi b}{l}\right) \cos \left(\frac{p \pi b}{l}\right)-\sin \left(\frac{m \pi a}{l}\right) \cos \left(\frac{p \pi a}{l}\right)\right\} \\
+m\left\{\sin \left(\frac{p \pi b}{l}\right) \cos \left(\frac{m \pi b}{l}\right)-\sin \left(\frac{p \pi a}{l}\right) \cos \left(\frac{m \pi a}{l}\right)\right\}
\end{array}\right] \\
+2 D_{2} \frac{\pi^{3}}{l^{3}} \sum^{A_{m}} m^{3}\left[\sin \left(\frac{p \pi a}{l}\right) \cos \left(\frac{m \pi a}{l}\right)-\sin \left(\frac{p \pi b}{l}\right) \cos \left(\frac{m \pi b}{l}\right)\right]= \\
-\rho_{2} h_{2} \omega^{2} \sum^{A_{m}}\left[\left\{\frac{\sin \left(\frac{(m-p) \pi b}{l}\right)-\sin \left(\frac{(m-p) \pi a}{l}\right)}{2 \pi(m-p)}\right\}-\left\{\frac{\sin \left(\frac{(m+p) \pi b}{l}\right)-\sin \left(\frac{(m+p) \pi a}{l}\right)}{2 \pi(m+p)}\right\}\right] \text { when } m \neq p ; \\
-D_{1} \frac{\pi^{4}}{l^{4}}\left(\frac{l}{2}\right) \sum A_{m} m^{4}-D_{2} \frac{\pi^{4}}{l^{4}} \sum\left[A_{m} m^{4}\left\{\frac{b-a}{2}-\frac{\sin \left(\frac{2 m \pi b}{l}\right)-\sin \left(\frac{2 m \pi a}{l}\right)}{4 m \pi} l\right\}\right] \\
-D_{2} \frac{\pi^{3}}{l^{3}} \sum^{A_{m}} m^{3}\left[\sin \left(\frac{2 m \pi b}{l}\right)-\sin \left(\frac{2 m \pi a}{l}\right)\right] \\
+D_{2} \frac{\pi^{3}}{l^{2}} \sum^{A_{m}} m^{3}\left[\sin \left(\frac{2 m \pi b}{l}\right)-\sin \left(\frac{2 m \pi a}{l}\right)\right] \\
=-\rho_{1} h_{1} \omega^{2} \frac{l}{2}-\rho_{2} h_{2} \omega^{2} \sum^{A_{m}}\left[\left\{\frac{b-a}{2}-\frac{\sin \left(\frac{2 m \pi b}{l}\right)-\sin \left(\frac{2 m \pi a}{l}\right)}{4 m \pi} l\right\}\right] \text { when } m=p . \quad(15)
\end{gathered}
$$

$$
\frac{\partial^{2} M_{x}}{\partial x^{2}}=\rho h \frac{\partial^{2} \omega}{\partial t^{2}}-R_{2} F_{o}
$$

where $R_{2}$ is the location function of applied force $F_{o}$, which can be expressed in terms of the Heviside function as:

$$
R_{2}= \begin{cases}0 ; & \text { when } \quad x<c \text { or } x>d \\ 1 ; & \text { when } \quad c \leq x \leq d\end{cases}
$$

where $M_{x}$ from equation Eq. (10) can be written as:

$$
\begin{gathered}
M_{x}=-D_{1} \frac{\partial^{2} \omega}{\partial x^{2}} \\
-D_{3} R_{1} \frac{\partial^{2} \omega}{\partial x^{2}}+\frac{R_{1} e_{13} V_{o}}{8 h_{2}}\left[\left(h_{1}+2 h_{2}\right)^{2}-h_{1}^{2}\right] ; \\
\frac{\partial M_{x}}{\partial x}=-D_{1} \frac{\partial^{3} \omega}{\partial x^{3}} \\
\quad-D_{3}\left[R_{1} \frac{\partial^{3} \omega}{\partial x^{3}}+\frac{\partial R_{1}}{\partial x} \frac{\partial^{2} \omega}{\partial x^{2}}\right]+D_{4} \frac{\partial R_{1}}{\partial x} ; \\
\frac{\partial^{2} M_{x}}{\partial x^{2}}=-D_{1} \frac{\partial^{4} \omega}{\partial x^{4}} \\
-D_{3}\left[R_{1} \frac{\partial^{4} \omega}{\partial x^{4}}+2 \frac{\partial R_{1}}{\partial x} \frac{\partial^{3} \omega}{\partial x^{3}}+\frac{\partial^{2} R_{1}}{\partial x^{2}} \frac{\partial^{2} \omega}{\partial x^{2}}\right]+D_{4} \frac{\partial^{2} R_{1}}{\partial x^{2}}
\end{gathered}
$$

where, $D_{1}=\frac{E_{1} h_{1}{ }^{3}}{12} ; D_{3}=\frac{E_{2}\left[\left(h_{1}+2 h_{2}\right)^{3}-h_{1}{ }^{3}\right]}{24} ; D_{4}=$ $\frac{e_{13} V_{0}\left[\left(h_{1}+2 h_{2}\right)^{2}-h_{1}^{2}\right]}{\left.8 h_{2}\right]}$.
Under the action of a harmonic force applied on the surface of the patch, the frequency of the voltage developed and transverse displacement of the beam will also be same. The expression of force, voltage, and displacement are: $F=F_{o} e^{i \omega t} ; V=$ $V_{o} e^{i \omega t} ; \omega=\sum A_{m} \sin \left(\frac{m \pi x}{l}\right) e^{i \omega t}$. Substituting these expressions in the equation Eq. (16), the equation of motion is obtained as:

$$
\begin{array}{r}
{\left[-D_{1} \frac{\pi^{4}}{l^{4}}-D_{2} R_{1} \frac{\pi^{4}}{l^{4}}\right] \sum^{A_{m}} m^{4} \sin \left(\frac{m \pi x}{l}\right)} \\
+D_{3} V_{o} \frac{\partial^{2} R_{1}}{\partial x^{2}}+2 D_{3} \frac{\pi^{3}}{l^{3}} \sum^{A_{m}} m^{3} \cos \left(\frac{m \pi x}{l}\right) \frac{\partial R_{1}}{\partial x} \\
+D_{3} \frac{\pi^{2}}{l^{2}} \sum^{A_{m}} m^{2} \sin \left(\frac{m \pi x}{l}\right) \frac{\partial^{2} R_{1}}{\partial x^{2}}+D_{4} \frac{\partial^{2} R_{1}}{\partial x^{2}}= \\
\omega^{2} \rho h \sum^{A_{m}} m \sin \left(\frac{m \pi x}{l}\right)-R_{2} F_{o} .
\end{array}
$$

The above equation is arranged in the matrix form as:

$$
\begin{aligned}
& {\left[\{K\}-\omega^{2}\{M\}\right] A_{m}-\{\theta\} V_{o}=} \\
& \quad F_{o} \frac{l}{m \pi}\left(\cos \frac{p \pi d}{l}-\cos \frac{p \pi c}{l}\right) ;
\end{aligned}
$$

where, $K=$ Stiffness matrix and $M=$ Mass matrix. Accounting for the damping, if present, Eq. (21) becomes:

$$
\begin{aligned}
& {\left[\{K\}+i \omega\{C\}-\omega^{2}\{M\}\right] A_{m}-\{\theta\} V_{o}=} \\
& F_{o} \frac{l}{m \pi}\left(\cos \frac{p \pi d}{l}-\cos \frac{p \pi c}{l}\right)
\end{aligned}
$$

where $C$ is damping coefficient. 
Now from equation Eq. (1), putting $E_{z}=V_{o} / R$, we get:

$$
D_{z}=e_{13} \varepsilon_{13}-p_{33} \frac{V_{o}}{h_{2}} .
$$

This can also be written as:

$$
D_{z}=-h_{2} e_{13} \frac{\partial^{2} \omega}{\partial x^{2}}-p_{33} \frac{V_{o}}{h_{2}} .
$$

The electric charge flow across the electrodes is given by

$$
q=\int_{a}^{b} D_{z} s d x=\int_{a}^{b}\left(-h_{2} e_{13} \frac{\partial^{2} \omega}{\partial x^{2}}-p_{33} \frac{V_{o}}{h_{2}}\right) s d x .
$$

Therefore, current $I$ is given as,

$$
I=\frac{d q}{d t}=\int_{a}^{b}-h_{2} e_{13} \frac{\partial^{3} \omega}{\partial x^{2} \partial t} s d x-C_{p} \frac{d V_{o}}{d t} ;
$$

here capacitance $C_{p}$ of the patch and is defined as:

$$
C_{p}=\frac{p_{33} s(b-a)}{h_{2}} .
$$

Therefore, from equation Eq. (23),

$$
\frac{V_{o}}{R}+C_{p} \frac{d V_{o}}{d t}=-\int_{a}^{b} h_{2} e_{13} \frac{\partial^{3} \omega}{\partial x^{2} \partial t} s d x
$$

Where, $R$ is the resistance of the energy harvesting circuit. Using the value of $\omega$ Eq. (24) can also be re-written as

$\frac{V_{o}}{R}+C_{p}(i \omega) V_{o}=\left(\frac{m \pi}{l}\right)^{2} \int_{a}^{b} h_{2} s e_{13}(i \omega) A_{m} \sin \left(\frac{m \pi x}{l}\right) d x$.

After integration, we get:

$$
\begin{aligned}
& \frac{V_{o}}{R}+C_{p}(i \omega) V_{o}= \\
& {\left[\cos \left(\frac{m \pi b}{l}\right)-\cos \left(\frac{m \pi b}{l}\right)\right]\left(\frac{m \pi}{l}\right) h_{2} s_{13}(i \omega) A_{m} .}
\end{aligned}
$$

Substituting the value of $A_{m}$ in Eq. (22), we get:

$$
\begin{aligned}
& \frac{\left\{[K]+i \omega[C]-\omega^{2}[M]\right\} V_{o}\left(\frac{1}{R}+i \omega C_{p}\right)}{\frac{s m \pi}{l} h_{2} e_{13} i \omega\left[\cos \left(\frac{m \pi b}{l}\right)-\cos \left(\frac{m \pi b}{l}\right)\right]}-[\theta] V_{o}= \\
& F_{o} \frac{l}{m \pi}\left(\cos \frac{p \pi d}{l}-\cos \frac{p \pi c}{l}\right) \text {. }
\end{aligned}
$$

The above equation can be rearranged to get $V_{o}$ as:

$$
V_{o}=\frac{i V_{1}}{V_{2}+i V_{3}}
$$

where,

$$
\begin{gathered}
V_{1}=F_{o} h_{2} e_{13} \omega\left[\cos \left(\frac{m \pi b}{l}\right)-\cos \left(\frac{m \pi b}{l}\right)\right] \\
\cdot\left[\cos \left(\frac{p \pi d}{l}\right)-\cos \left(\frac{p \pi c}{l}\right)\right] ; \\
V_{2}=\left\{[K]-\omega^{2}[M]\right\} \frac{1}{R}-\omega^{2}[C] C_{p} \\
V_{3}=\left\{[K]-\omega^{2}[M]\right\} \omega C_{p}+\frac{\omega[C]}{R} \\
-[\theta] \frac{s m \pi}{l} h_{2} e_{13} \omega\left[\cos \left(\frac{m \pi b}{l}\right)-\cos \left(\frac{m \pi b}{l}\right)\right] .
\end{gathered}
$$

The equation for the above circuit can be written as:

$$
\frac{\int^{i} d t}{C}+i R=V \sin \omega t
$$

We get the following equation in Laplace form:

$$
\begin{aligned}
\left(\frac{I(s)}{C s}\right)+I(s) R=\frac{V \omega}{s^{2}+\omega^{2}} & \\
I(s)\left(\frac{1+R C s}{C s}\right) & =\frac{V \omega}{s^{2}+\omega^{2}} ; \\
I(s) & =\frac{V C \omega s}{(1+R C s)\left(s^{2}+\omega^{2}\right)} .
\end{aligned}
$$

The voltage across resistor $R$ is:

$$
V_{o}(s)=I(s) R=V R C \omega\left[\frac{s}{(1+R C s)\left(s^{2}+\omega^{2}\right)}\right] .
$$

On inverse transformation of the Laplace equation, we get:

$$
V_{o}=\frac{-V R C \omega}{\left(1+R^{2} C^{2} \omega^{2}\right)}\left[e^{\frac{-t}{R C}}+\cos (\omega t)+R C \omega \sin (\omega t)\right] .
$$

The magnitude of the energy output $(P)$ from the vibration of a simply supported beam under the action of a harmonic force is given by:

$$
P=\frac{V_{o}^{2}}{R} .
$$

The Voltage across the load resistance is calculated analytically by solving Eq. (26) in MATLAB. The power across the load can be calculated using Eq. (28). The results are verified using COMSOL software. The software gives the voltage across the patch, which can be used to determine COMSOL voltage across the load using Eq. (27). The results obtained are discussed below.

\section{MODELLING WITH ANSYS AND COMSOL}

\subsection{ANSYS}

To model the beam in ANSYS12, we used BEAM 3 element and solved the 2-D beam problem. The real constants for the beam elements are given in terms of area $=2 \times 10^{-5} \mathrm{~m}^{2}$, moment of inertia $=1.667 \times 10^{-12} \mathrm{~m}^{4}$ and height $=1 \mathrm{~mm}$. The real constants for the patch of thickness $0.2 \mathrm{~mm}$ are given in terms of area $=4 \times 10^{-6} \mathrm{~m}^{2}$, moment of inertia $=1.45333 \times 10^{-12} \mathrm{~m}^{4}$ and height $=0.2 \mathrm{~mm}$. Here we consider the width of both patch and beam as $1 \mathrm{~cm}$. The material properties are considered as isotropic and are given in Table 1. The patch is constrained with the beam by coupling the degrees of freedom for both. The constraint applied at both of the two ends has a displacement of 0 and the moment is 0 , which satisfies the condition for simply supported beam. The mesh size is 200 elements over the whole length of the beam, i.e., 1 element per mm of beam length.

\subsection{COMSOL}

The beam is modeled in 3-D in COMSOL 4.3. The modal analysis is done by choosing the solid mechanics physics and 
Table 1. Comparison of Energy Density.4

\begin{tabular}{|c|c|c|}
\hline Type & Energy Density $(\mathrm{mJ} / \mathrm{cm} 3)$ & Assumption \\
\hline Piezoelectric & 35.4 & PZT 5 H \\
\hline Electromagnetic & 24.8 & $0.25 \mathrm{~T}$ \\
\hline Electrostatic & 4 & $3 \times 107 \mathrm{~V} \mathrm{~m}-1$ \\
\hline
\end{tabular}

Table 2. The material properties.

\begin{tabular}{|c|c|c|}
\hline Parameter & Host Beam & Patch (PZT) \\
\hline Density(kg/m3) & 2800 & 7800 \\
\hline Modulus of elasticity(N/m2) & $68.3 \mathrm{e} 9$ & $12 \mathrm{e} 10$ \\
\hline Poisson's ratio & 0.3 & 0.2 \\
\hline Piezoelectric constant e13 =e23(C/m2) & - & -5.2 \\
\hline Permittivity(p) & - & $1.5 \mathrm{e}-8$ \\
\hline
\end{tabular}

eigen frequency as the study. The constraint is applied with Rigid connectors at both the end faces to make the beam simply supported with the rotation constrained in two directions $R_{x}=0$ and $R_{z}=0$ and all the three displacement components as 0 . The mesh is generated using free tetrahedral elements and the setting size is extra fine. The solution is then computed for getting natural frequency, as tabulated in Table 3.

To carry out power calculations, transient analysis is done with COMSOL physics as piezoelectric devices and study as time-dependent. A boundary load, which is harmonic in nature $(=2500 \sin (w t) \mathrm{Pa})$, is applied on the top of piezoelectric patch and power across the top and bottom of the patch is computed when the load resistance is $1000 \Omega$. Again, the mesh is created using free tetrahedral elements with an extra fine size. The results from COMSOL are compared with results of our analytical model and are discussed in next section.

\section{RESULTS AND DISCUSSION}

Table 2 shows the material properties for the beam and the patch. Results for various cases have been taken, which have been divided in two sections. Equations (15)a and (15)b are solved through MATLAB program to get the natural frequency of the beam with the bonded patch. The results obtained were verified using ANSYS software package by modeling the beam with the bonded patch. The results obtained were in close agreement with the results of ANSYS hence validating the theoretical work. This is tabulated in Table 3.

Equations 26 and 28 are solved through MATLAB program to get the power extracted across the load resistance from the patched beam. The results obtained were verified using COMSOL software package by modeling the patched beam. The results obtained were in close agreement with the results of COMSOL, hence validating the theoretical work.

Table 3. Result validation.

\begin{tabular}{|c|c|c|c|}
\hline $\begin{array}{c}\text { Mode } \\
\text { of Frequency }\end{array}$ & $\begin{array}{c}\text { Theoretical } \\
\text { Frequency (Hz) }\end{array}$ & $\begin{array}{c}\text { ANSYS (Hz) } \\
\text { Frequency (Hz) }\end{array}$ & $\begin{array}{c}\text { COMSOL (Hz) } \\
\text { Frequency (Hz) }\end{array}$ \\
\hline 1 & 56.821 & 56.75 & 56.95 \\
\hline 2 & 224.028 & 223.99 & 229.96 \\
\hline 3 & 518.743 & 518.00 & 520.27 \\
\hline 4 & 897.412 & 896.83 & 924.75 \\
\hline 5 & 1450.408 & 1448.40 & 1462.38 \\
\hline
\end{tabular}

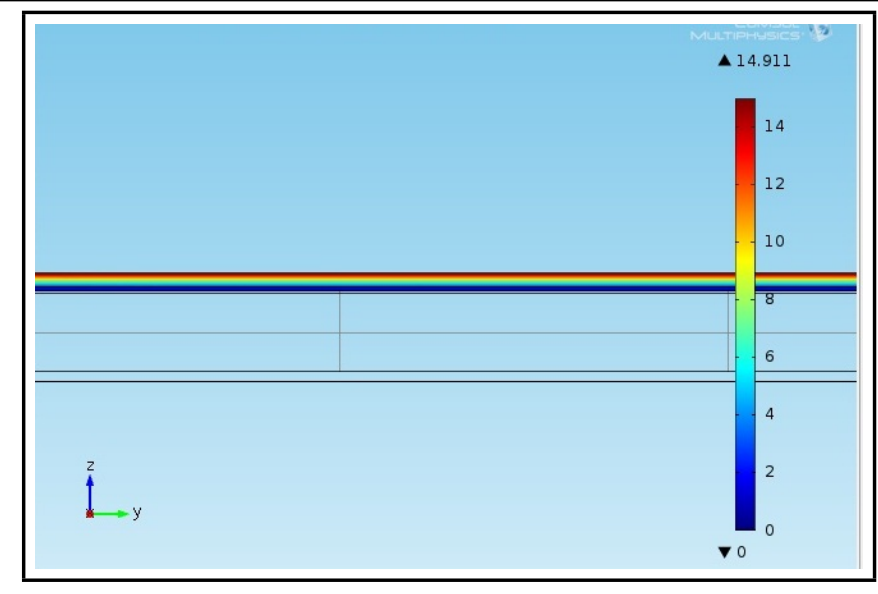

Figure 4. Voltage across the PZT patch.

The PZT patch with thickness $0.2 \mathrm{~mm}$ and length $20 \mathrm{~mm}$ is placed $90 \mathrm{~mm}$ from the support in a $200 \mathrm{~mm}$ long simply supported beam. From the analytical model, the potential difference across the beam is found to be $14.248 \mathrm{~V}$ while from COMSOL model, it is found to be 14.911 V (see Fig. 4). Similarly, the voltage across the load resistance of $100 \mathrm{k} \Omega$ is found analytically to be $7.1072 \mathrm{~V}$; while using COMSOL, it is found to be $7.438 \mathrm{~V}$. The slight difference is due to the reason that there would be some damping due to dissipation of heat across the load, which has not been taken into account in COMSOL model.

\subsection{Parametric Study}

This section deals with a parametric study of several variables in order to determine how the natural frequency and power are affected by the variables. After a certain thickness, the natural frequency starts decreasing for all modes because of the dominance of the mass and the stiffness of the patch in comparison to stiffness contribution by the host beam because of the electrical properties (see Fig. 5). As depicted from the moment and force equations, electrical properties contribute towards stiffness of the plate which results in increase in the frequency for all the modes (see Fig. 6). The natural frequency increases as the length of the patch increases. The location of the patch also affects the natural frequency of the combination, as shown in Fig. 7. The placement of piezoelectric patch at the centre of the beam is the maximum displacement position for the first mode which in turn gives more contribution to stiffness.

For energy harvesting, the PZT length and thickness, along with the beam thickness, has been studied. The position of the external forcing function will be optimized for maximum output as well. To calculate the energy output one has to do free vibration analysis first in order to evaluate the fundamental frequencies and the results are used in the calculations of energy harvesting. Figures 8,9 , and 10 show the maximum energy harvested $\left(P_{\max }\right)$ for patch of thickness $0.2 \mathrm{~mm}$ and $0.5 \mathrm{~mm}$ respectively against the load resistance for various areas. This means that the kinetic energy input due to external excitation remains constant whereas the energy harvested varies with the PZT patch length, patch thickness and with the resistance of energy harvesting circuit. 


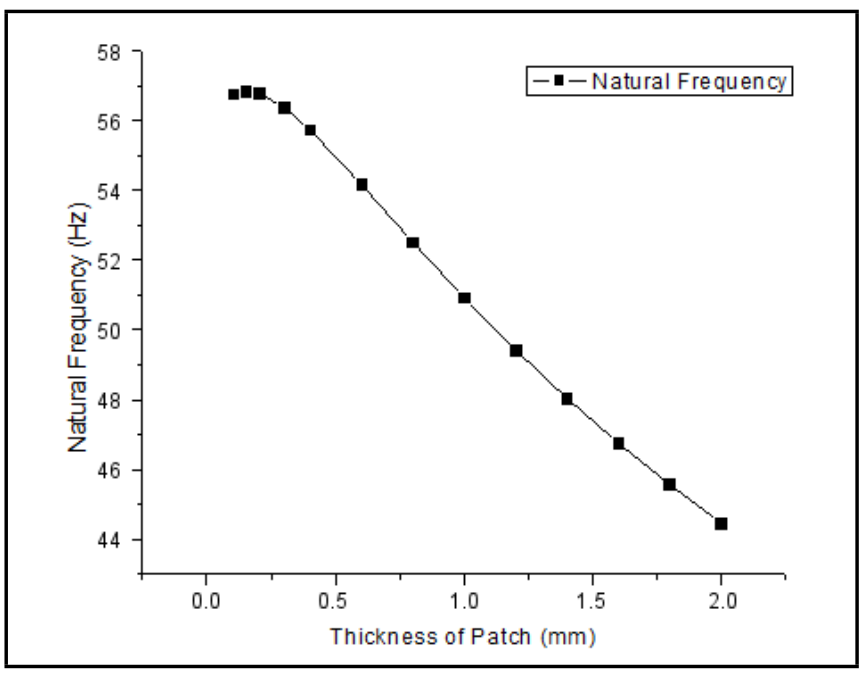

Figure 5. Effect of Patch Thickness on Natural Frequency $(a=90 \mathrm{~mm}$ and $b-$ $\mathrm{a}=2 \mathrm{~mm})$.

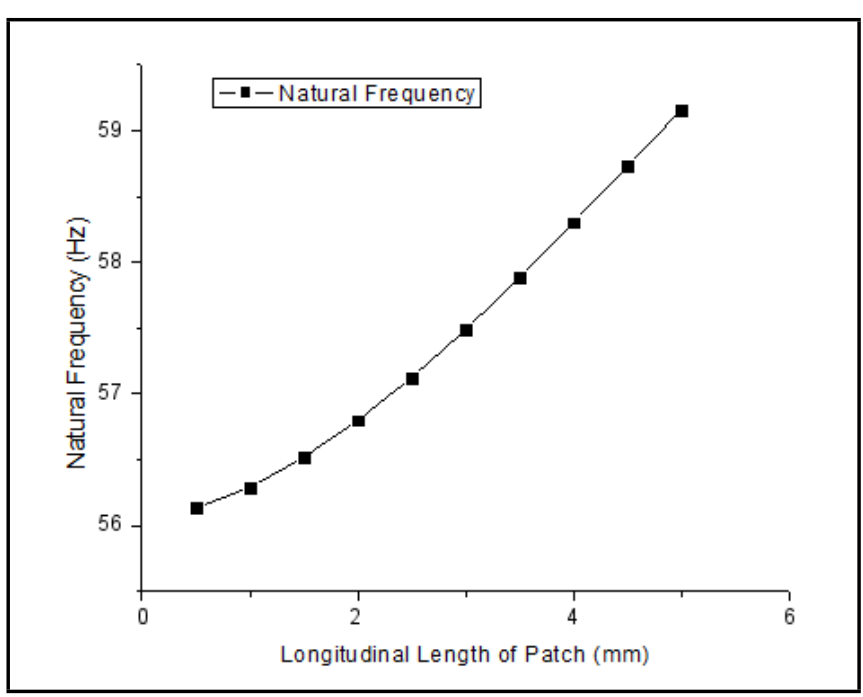

Figure 6. Effect of Patch Length on the Natural Frequency $(\mathrm{h} 2=0.2 \mathrm{~mm}$ and $\mathrm{a}=90 \mathrm{~mm})$.

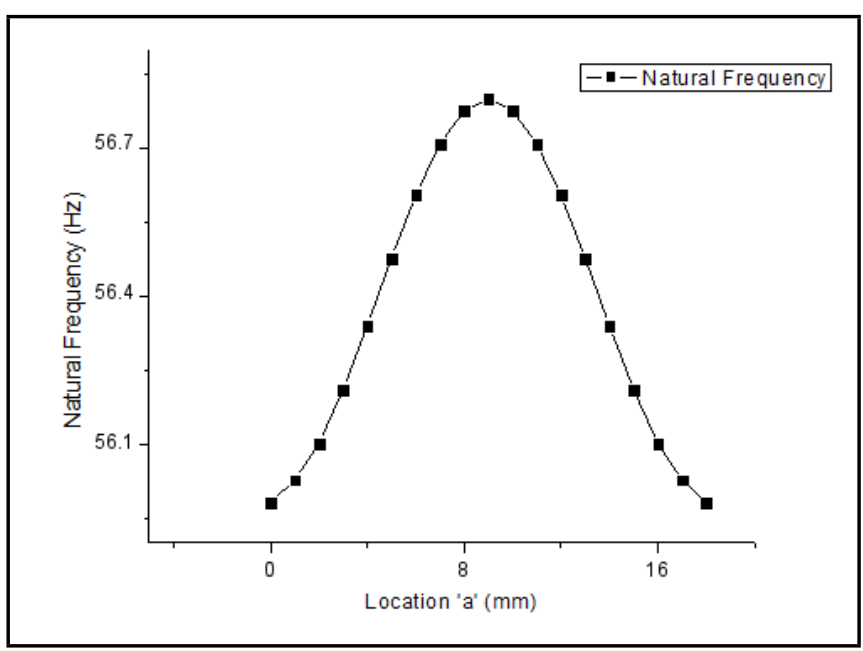

Figure 7. Effect of Patch Location on Natural Frequency (h2 $=0.2 \mathrm{~mm}$ and $\mathrm{b}-\mathrm{a}=20 \mathrm{~mm})$

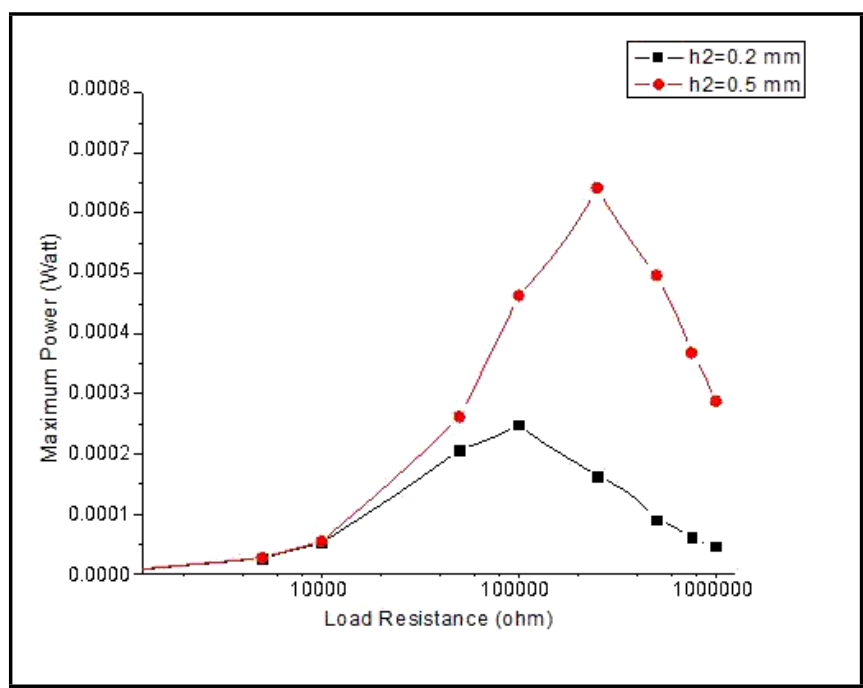

Figure 8. Power with Rl at different patch thicknesses.

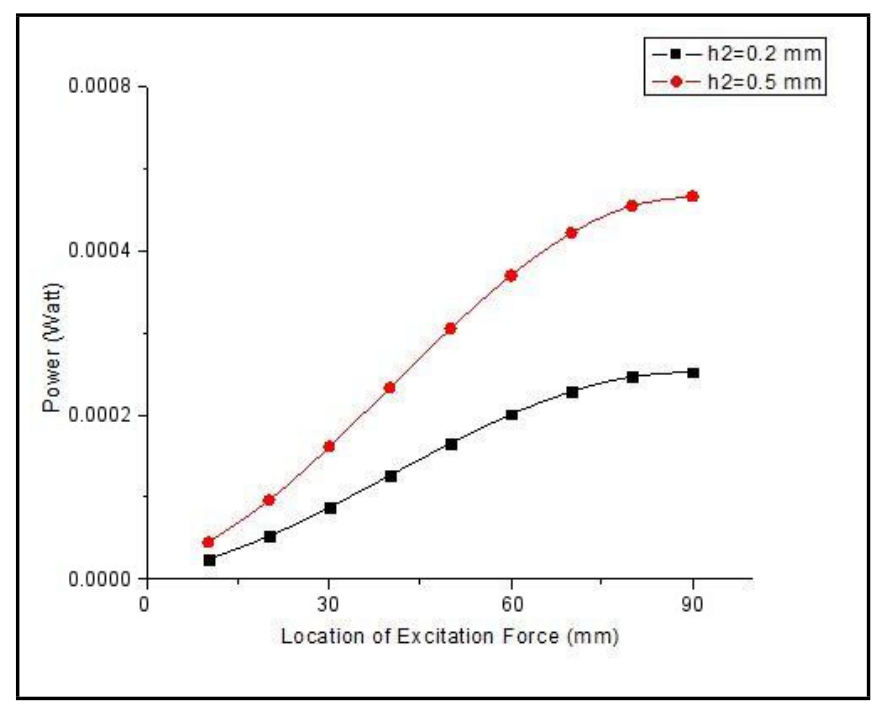

Figure 9. Power with different locations of the excitation force at different patch thicknesses.

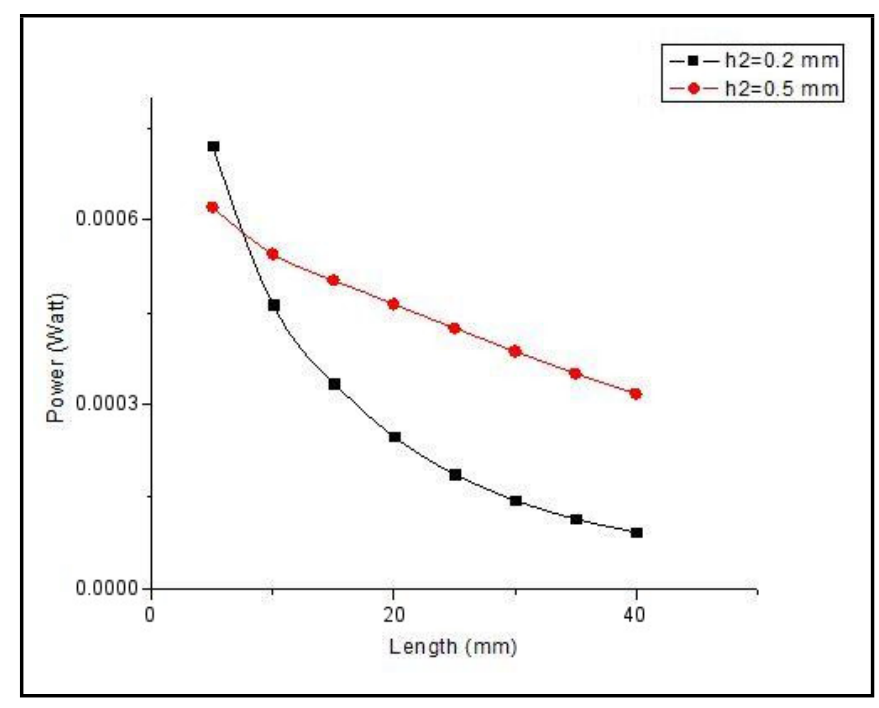

Figure 10. Power with length of patch at different patch thicknesses. 
As the PZT length increases, it starts to affect the overall characteristics of the beam system, changing the effective cross-section, Young's modulus, and natural frequencies. This will have adverse effects, reducing the beam deflections, strain experienced by the PZT, and overall power produced. ${ }^{10}$

The farther away the forcing function is from the beam's hinged end, the larger the moment applied to the beam through force. So, it follows that the optimal location for the force is at the centre of the beam, which creates the largest moment.

\section{CONCLUSIONS}

The above study is carried out with the help of the generalized Hooke's Law and by considering electrical properties of the PZT. Theoretical analysis went forward from EulerBernoulli Beam Theory and moment equation. The results obtained are verified with the help of MATLAB, ANSYS, and COMSOL. Similar study can be carried out for the cantilever beam, as it will generate more power output but in the cost of reduced safety factor.

The effect of electrical properties of the patch on the transverse vibration of a simply supported beam with PZT patch has been investigated for varying thickness and for different positions taken by the patch. The formulation and analytical determination of the coupling effects were demonstrated to be related to modal frequencies. Electrical properties significantly affected the response of transverse vibration frequencies. As the length of piezoelectric patch increased, an increase in the modal frequency was achieved experimentally.

Similarly, the parametric study with various patch thickness, patch length, load resistance and location of excitation force was done. It was found that the energy harvested varies with the PZT patch length, patch thickness and with the resistance of energy harvesting circuit.

Some of the unsolved problems with this project are assumption of isotropy in the analysis part and accumulation of continuous power generated by PEG. Piezoelectric material is not isotropic, and there might be some numerical differences if an anisotropic analysis is used. The stiffness differs slightly in different directions, and the Poisson's ratio differs by a very small amount. It is unlikely that this anisotropic problem causes a major difference in the power generation from the above analysis with an isotropic patch assumption, but there is an argument for carrying out the analysis.

\section{REFERENCES}

1 Roundy, S., Wright, P. K., and Rabaey, J. , A study of low level vibrations as a power source for wireless sensor nodes, Computer Communications, 26, 1131-1144, (2003). https://dx.doi.org/10.1016/S0140-3664(02)00248-7
2 Williams, C. B., and Yates, R. B., Analysis of a micro-electric generator for Microsystems, Stockholm, Sweden, (1995), 369-372. https://dx.doi.org/10.1109/sensor.1995.717207

3 Roundy, S., Wright, P. K., and Pister, K. S. J., Micro-electrostatic vibration-to-electricity converters, New Orleans, LA, United States, (2002), 487-496. https://dx.doi.org/10.1115/IMECE2002-39309

4 Roundy, S. and Wright, P.K., A piezoelectric vibration based generator for wireless electronics, Smart Materials and Structures, 13, 1131-1142, (2004). https://dx.doi.org/10.1088/0964-1726/13/5/018

5 Tyagi, A. and Ghosh, M. K., Modal analysis of transverse vibrations of a thin rectangular plate with a bonded piezoelectric patch, Int. J. Vehicle Structures and Systems, 3 (4), 234-240, (2011). https://dx.doi.org/10.4273/ijvss.3.4.04

6 Sodano H. A., Inman D. J., and Park G., A review of power harvesting from vibration using piezoelectric materials, The Shock and Vibration Digest, 36(3), 197-205, (2004). https://dx.doi.org/10.1177/0583102404043275

7 Sodano H. A., Park G., and Inman D. J., Estimation of electric charge output for piezoelectric energy harvesting, Strain, 40, 49-58, (2004). https://dx.doi.org/10.1111/j.1475-1305.2004.00120.x

8 Chen S. N., Wang G. J., and Chien M. C., Analytical modeling of piezoelectric vibration-induced micro power generator, Mechatronics, 16, 379-387, (2006). https://dx.doi.org/10.1016/j.mechatronics.2006.03.003

9 Priya, S., Advances in energy harvesting using low profile piezoelectric transducers, J. Electroceram, 19, 165-182, (2007). https://dx.doi.org/10.1007/s10832-007-9043-4

10 Erturk A. and Inman D. J., A distributed parameter electromechanical model for cantilevered piezoelectric energy harvesters, Journal of Vibration and Acoustics, 130 / 041002, 1-15, (August 2008). https://dx.doi.org/10.1115/1.2890402

11 Xu, C., Liang, Z., Ren, B., Di, W., Luo, H., Wang, D., Wang, K., and Chen, Z. Bi-stable energy harvesting based on a simply supported piezoelectric buckled beam, Journal of Applied Physics, 114, 114507, (2013). https://dx.doi.org/10.1063/1.4821644

12 Erturk A. and Inman D. J., An experimentally validated bimorph cantilever model for piezoelectric energy harvesting from base excitations, Smart Mater. Struct., 18 (025009), 1-18, (2009). https://dx.doi.org/10.1088/09641726/18/2/025009 